\title{
Lesiones inducidas por contacto directo con antenas de radio en vehículos de Caballería
}

\author{
Díaz de Tuesta Revilla I. ${ }^{1}$
}

Sanid. mil. 2014; 70 (3): 188-190; ISSN: 1887-8571

\begin{abstract}
RESUMEN
La exposición sostenida a emisiones de radio podría estar relacionada con lesiones en el tejido subcutáneo. Presentamos dos casos sucedidos en tiradores de vehículo explorador de Caballería (VEC) tras unas maniobras en zona de operaciones Líbano, consistentes en una inusual tumefacción del tejido adiposo en antebrazo derecho sin antecedente de contusiones, ni contactos químicos o irradiación conocida, y con piel íntegra, que coincide con zonas de posible contacto mantenido con la antena del vehículo. La causalidad no es determinante por tratarse de una relación observacional, pero los hallazgos sugieren que deberían tomarse medidas en relación con la reducción del riesgo de exposición a radiaciones electromagnéticas en operarios de carros con antenas plegables.
\end{abstract}

PALABRAS CLAVE: Grasa subcutánea, Diatermia, Ondas de radio, Personal militar. Radiación.

Injuries induced by direct contact with Cavalry vehicles radio antennas

Sustained exposure to radio emissions could be related to injuries in the subcutaneous tissue. We present two cases registered in shooters of Cavalry Exploration Vehicle (VEC) after maneuvers in Lebanon operation area, consisting of an unusual swelling of adipose tissue in right forearm, with no known history of contusions, chemical contacts or irradiation, and with preserved skin integrity, which coincides with areas of possible contact with the antenna of the vehicle. Since the previous relation is only observational, causality is not decisive, but the findings suggest that measures should be taken in operators of cars with folding antennas, in order to reduce the risk of exposure to electromagnetic radiation..

KEY WORDS: Subcutaneous fat, Diathermy, Radio waves, Military personnel, Radiation.

\section{INTRODUCCIÓN}

Las comunicaciones por radio forman parte esencial de la actividad de la Fuerza. Exceptuando las emisiones de SHF de los enlaces de microondas, se considera que en términos normales las comunicaciones por radio entre unidades no constituyen un riesgo relevante para la salud ${ }^{1}$.

En Agosto de 2013 se desarrolló en Líbano el ejercicio de tiro "Steel Storm", consistente en una prueba de armamento de las fuerzas españolas destacadas en la Base Miguel de Cervantes en Ebel Es Saqi, Líbano. Participaron entre otros tres Vehículos Exploradores de Caballería (VEC) que realizaron ejercicios rutinarios de tiro con armamento 12 '70 $\mathrm{mm}$ y $25 \mathrm{~mm}$.

Tras el ejercicio se produjeron dos incidentes que nos hacen pensar que en la configuración actual, determinada combinación de factores pueden generar lesiones inflamatorias por radiofrecuencia en personal de los carros.

${ }^{1}$ Tte. Médico (RV). Role 1 - BRILIB XIX, Ebel Es Saqi - Marjayoun Líbano.

Dirección para correspondencia: Ignacio Díaz de Tuesta Revilla. Hospital Universitario La Paz. Servicio de Cirugía Cardiovascular. Paseo de La Castellana 261. 28046 Madrid, España.reserva@tuesta.ne

Recibido: 22 de noviembre de 2013

Aceptado: 23 de enero de 2014

\section{OBSERVACIONES CLÍNICAS}

El día siguiente al ejercicio acudió a consulta (Role 1) el tirador de uno de los VEC implicados en las maniobras, con una lesión atípica en tercio distal del antebrazo derecho. Los síntomas se iniciaron 6 horas después de la actividad. Presentaba dolor moderado al contraer la musculatura extensora de dedos y supinadora del antebrazo, y tumefacción en el dorso del antebrazo. La piel estaba íntegra, sin signos de quemaduras, irritaciones por contacto ni picaduras de insectos. Por ecografía se observó una inflamación del tejido subcutáneo con ligero edema, aumento de la distancia entre piel y fascia muscular respecto al miembro contralateral, y trombosis no oclusiva de vena cefálica en su trayecto por la zona edematizada.

El paciente no identificó ningún evento desencadenante. No recordaba contusiones, maniobras bruscas o carga de pesos fuera de lo común. No hubo contactos químicos sospechosos, mordeduras o punciones. Según refería, su labor durante el ejercicio fue similar al de otros muchos realizados en territorio nacional, sin que en ocasiones anteriores o patrullas en zona hubiese padecido molestias similares. El cuadro fue interpretado como una dudosa trombosis de vena cefálica con posible edema secundario desproporcionado al esperado, y fue tratado con antiinflamatorios locales y sistémicos.

Pocas horas más tarde acudió el tirador del segundo VEC con una lesión de características similares al anterior, de extensión más amplia, y también con un dolor moderado, menor del esperado para el grado de inflamación observado si esta fuera 
por contusión o infección, que se había iniciado a las 12 horas de la maniobra. La lesión también se localizaba en la cara dorsal del antebrazo derecho, solo que en esta ocasión la zona afectada era el tercio proximal y el codo (Figura 1), con molestias a la contracción de la musculatura subyacente. La piel estaba íntegra, no había hematomas, rubor, pérdidas de vello o lesiones cutáneas. Como en el caso previo el tirador no identificó desencadenantes. Los hallazgos ecográficos fueron superponibles al caso anterior: inflamación y edema del tejido subcutáneo. En este caso se trataba de una zona avascular sin venas superficiales relevantes.

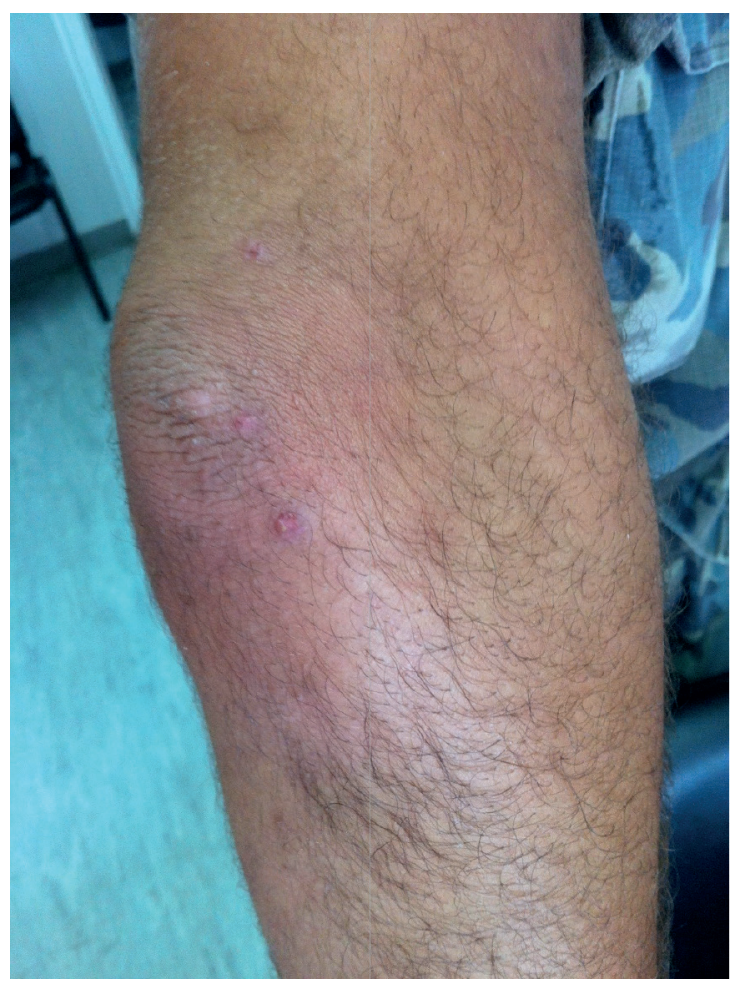

Figura 1. Inflamación del codo del segundo tirador.

La coincidencia temporal de ambos casos con hallazgos poco frecuentes, sin desencadenante reconocible ni lesiones tróficas en la piel, nos llevó a indagar causas ambientales relacionadas con la actividad del día anterior, porque los dos afectados ocupaban la misma posición en sus respectivos VEC.

El tiro se realiza desde el interior del blindado. Reproducida esta actividad no identificamos fuentes de calor o relieves en su zona de movimientos que pudieran producir microtraumatismos o exposiciones electromagnéticas relevantes. Tampoco parecía evidente el contacto inadvertido con substancias químicas. Las torretas cuentan con emisoras de radio, pero están situadas detrás de los asientos y equidistantes al jefe de carro, que no resultó afectado en ninguno de los dos casos. La versión de VEC empleada dispone de mira de infrarrojos, pero es un receptor pasivo, no un emisor. No dispone de dispositivos láser ni otros elementos emisores de radiaciones.

Durante el movimiento del vehículo el tirador se mantiene en pie sobre su asiento con el tórax fuera de la torreta para misión de vigilancia. En esta posición las antenas de los inhibido- res de frecuencia quedan tras él, a unos $50 \mathrm{~cm}$. Los inhibidores están protegidos por una rejilla contra la que es posible recibir golpes, pero serían en la parte dorsal del brazo y difícilmente pasarían inadvertidos.

Los carros disponen de dos antenas de comunicaciones encastradas en la parte trasera de la torreta. En modo normal de operación estas antenas se mantienen verticales para asegurar el máximo alcance. Esta configuración eficiente para el campo libre es poco práctica para el tráfico rodado en zona de operaciones, porque se golpea con techos, señalizaciones y construcciones elevadas. Por ello es habitual mantenerlas dobladas con la punta amarrada a la parte anterior de la torreta, describiendo un arco a escasos centímetros del observador, en su zona natural de apoyo (Figura 2).

Consultados los tiradores, ambos reconocieron haber circulado con el antebrazo apoyado en la antena, con el añadido de que el tirador que presentaba la tumefacción cerca del codo circula mirando hacia delante, mientras que el tirador del segundo carro relató que habitualmente realiza el viaje observando el perímetro de su lado del vehículo, girado hacia la derecha, de forma que la parte en la que se apoya de forma natural en la antena corresponde a la cercana a la mano.

La maniobra en cuestión se realizó en un campo a 2:30h de la base. Revisadas las características de los desplazamientos, por cuestiones operativas la radio no funcionó en el modo habitual de salto de frecuencia, sino en frecuencia fija de $47^{\prime} 850 \mathrm{MHz}$. Además, por la orografía del terreno que dificulta la comunicación en banda VHF, se trabajó en el segmento más alto de emisión: 50W. Finalmente, los operarios recuerdan el día como especialmente denso en comunicaciones radio.

Respecto a las lesiones de los dos afectados, las tumefacciones remitieron a los pocos días con tratamiento antiinflamatorio convencional, sin secuelas aparentes y sin limitaciones de movilidad o dolor.

\section{DISCUSIÓN}

La radiación no ionizante de alta energía como los enlaces de microondas (bandas SHF y EHF) son causa reconocida de lesiones si se produce una exposición mantenida. Explotando este efecto se han desarrollado armas no letales como el "Active Denial System" que desplegó el ejército norteamericano en Afganistán, consistente en una antena direccional de microondas montada sobre vehículo capaz de dirigir el haz contra posibles amenazas para dispersarlas por el disconfort que produce ${ }^{2}$.

Las radiofrecuencias de menor energía tienen utilidad terapéutica. Son la base de bisturís eléctricos, procedimientos percutáneos como la lipolisis ${ }^{3}$, procedimientos endovasculares como el tratamiento de varices ${ }^{4}$ y la denervación renal ${ }^{5}$, y procedimientos percutáneos como la denervación radicular ${ }^{6}$.

Exceptuando estos usos, diversos estudios acreditan que los equipos de radiofrecuencia empleados para comunicaciones que trabajan en las bandas UHF y VHF no causan interacciones biológicas significativas ${ }^{7}$. Los equipos se estudian para minimizar la absorción de radiación en condiciones normales de uso ${ }^{8}$.

Sin embargo, hay circunstancias en las que una combinación de factores puede superar los márgenes de seguridad 


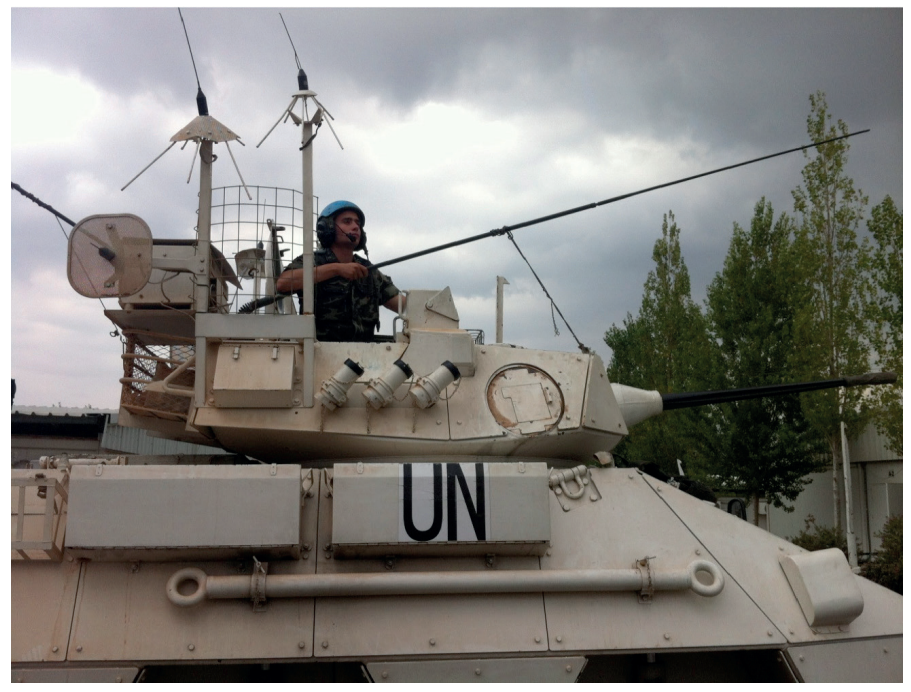

Figura 2. Vehículo Explorador de Caballería.

previstos, y este parece ser uno de ellos. En nuestro caso los condicionantes locales hicieron que una antena diseñada para funcionar en posición vertical a suficiente distancia de la tripulación, se convirtiese en un apoyabrazos natural, incrementando en varios órdenes de magnitud la radiación recibida. Probablemente no habría tenido mayores consecuencias de no darse otros factores potenciadores.

Las transmisiones entre unidades suelen hacerse a una frecuencia que cambia continuamente en un rango de $30 \mathrm{MHz}$ a 90 $\mathrm{MHz}$ para dificultar la intercepción por radioescuchas no autorizadas. En este caso por cuestiones tácticas no se empleó salto de frecuencia sino que se emitió continuamente a una frecuencia próxima a $40 \mathrm{MHz}$. Esta frecuencia es similar a la de máxima absorción de energía para una persona de pie conectada a tierra, que es $30 \mathrm{MHz}^{9}$. Es posible que un salto de frecuencia sea más tolerable tanto por alejarse de esta frecuencia, como porque el tiempo en el que cada tejido es expuesto a la frecuencia que más lo excita es menor, favoreciendo la dispersión del calor generado entre distintas capas.

A esto se suma la energía emitida. Una inusitada actividad de comunicaciones para coordinar un convoy de larga duración en terreno en el que no prima el "silencio radio", con una topografía abrupta en la que fue necesaria la potencia máxima de emisión $(50 \mathrm{~W})$ fueron posiblemente los factores finales que junto con la frecuencia fija y el contacto directo con la antena generaron un calentamiento continuado del tejido adiposo.

Por sus características, este tipo de emisión eleva unos pocos grados del tejido adiposo, poco perceptible porque se genera en profundidad fuera del alcance de los termoreceptores cutáneos, de ahí precisamente su tolerancia e interés en tratamientos estéticos. Al producirse este fenómeno en un entorno no contro- lado, el calentamiento pudo superar los márgenes terapéuticos induciendo la inflamación observada en los tiradores.

Este fenómeno es inhabitual, y de hecho habría pasado probablemente desapercibido de no haberse dado la coincidencia de dos casos. Como consecuencia de las observaciones, emitimos una recomendación consistente en:

Evitar el contacto con la antena. Una orden de "prohibido apoyarse" parece poco realista. En un traslado prolongado y penoso, es difícil creer que pueda evitarse utilizar lo que constituye un apoyabrazos natural. La solución ergonómica sería situar la antena lo suficientemente lejos para que no resulte "inevitable" apoyarse en ella.

Intentar emitir en salto de frecuencia, no solo por razones tácticas, sino para dispersar la absorción proporcional de energía emitida.

En la medida de lo posible, emitir con la menor potencia posible. No solo para reducir la posibilidad de ser detectado, algo bien conocido en el ámbito militar, sino porque se minimizan los efectos biológicos de las radiofrecuencias.

Finalmente, en lo que a sanidad militar respecta, debería tenerse en cuenta este mecanismo ante inflamaciones desproporcionadas al nivel de dolor sin causa aparente, en profesionales que trabajen cerca de radioemisores.

\section{AGRADECIMIENTOS}

Por su colaboración en este estudio al Tte. Garcia-Cuadrado I.

\section{BIBLIOGRAFÍA}

1. Vargas F, Úbeda A. Campos electromagnéticos y salud pública. Informe técnico elaborado por el comité de expertos. Dirección general de salud pública y consumo, Subdirección general de sanidad ambiental y salud laboral; 2001.

2. U.S. Department of Defense. Non-Lethal Weapons Program. [Online]. [cited 201308 18. Available from: HYPERLINK http://jnlwp.defense.gov/pressroom/adt.htm

3. Paul M, Mulholland R. A new approach for adipose tissue treatment and body contouring using radiofrequency-assisted liposuction. Aesthetic Plast Surg. 2009 Sept; 33(5):687-94.

4. Gohel MS, Davies AH. Radiofrequency ablation for uncomplicated varicose veins. Phlebology. 2009; 24 Suppl 1:42-9.

5. Barbash IM, Waksman R. Sympathetic renal denervation: Hypertension beyond SYMPLICITY. Cardiovasc Revasc Med. 2013; 14(4):229-35.

6. van Boxem K, van Eerdm, Brinkhuizen T, Pati L. Radiofrequency and pulsed radiofrequency treatment of chronic pain syndromes: the available evidence. Pain Pract. 2008 Sept-Oct; 8(5):385-93.

7. Roberts NJ, Michaelson SM. Epidemiological studies of human exposure to radiofrequency radiation: A critical review. Int Arch Occup Environ. 1985; 56:169-178.

8. IEEE Std (IEEE). «Standard for Safety Level with Respect to Human Exposure to Radio Frequency Electromagnetic Fields, $3 \mathrm{KHz}$ to $300 \mathrm{GHz}$ ». ; Oct 2005. Report No.: C95.1.

9. Knave B. Radiaciones no ionizantes. En Enciclopedia de Salud y Seguridad en el Trabajo. 4th ed.: Organización Internacional del Trabajo; 1998. 